\title{
The efficacy of empiric arterial embolization for treating severe hemoptysis
}

\author{
Peter I. Kalmar ${ }^{1}$, Peter Schedlbauer ${ }^{1}$, Martin Wehrschütz ${ }^{1}$, Otto C. Dörfler ${ }^{1}$, \\ Hannes A. Deutschmann ${ }^{1}$, Freyja-Maria Smolle-Jüttner ${ }^{2}$, Rupert H. Portugaller ${ }^{1}$ \\ ${ }^{1}$ Medical University of Graz, Department of Radiology, Division of Vascular and Interventional Radiology, University Hospital Graz, \\ Graz, Austria \\ ${ }^{2}$ Medical University of Graz, Department of Surgery, Division of Thoracic and Hyperbaric Surgery, University Hospital Graz, Graz, \\ Austria \\ Email: peter.schedlbauer@klinikum-graz.at,martin.wehrschuetz@klinikum-graz.at,otto.doerfler@klinikum-graz.at, \\ hannes.deutschmann@medunigraz.at, freyja.smolle@medunigraz.at, rupert.portugaller@medunigraz.at
}

Received 23 March 2013; revised 23 April 2013; accepted 1 May 2013

Copyright (C) 2013 Peter I. Kalmar et al. This is an open access article distributed under the Creative Commons Attribution License, which permits unrestricted use, distribution, and reproduction in any medium, provided the original work is properly cited.

\begin{abstract}
Aim: Purpose of the study was to investigate the efficacy of empiric arterial embolization and to achieve hemostasis in patients with massive hemoptysis. Materials and Methods: A retrospective review of histories and interventional studies of 56 patients (40 male, 16 female, median age 57 years; range, 16 - 83 years) referred endovascular treatment of massive hemoptysis over a period of $\mathbf{1 7}$ years. Arteries supposed to supply the bleeding bronchoalveolar sections were embolized with particles in all cases. Digital subtraction angiographical (DSA) studies were analyzed with respect to the morphology of the embolized arteries. Arteries were termed pathologic when they were either hypertrophic or supplied hypervascular lung sections as well as actively bleeding branches. Empiric embolization was defined as endovascular occlusion of arteries without visible contrast-material extravasation on DSA studies. Results: Continuing hemoptysis was encountered in one (25\%) of 4 patients with active contrast extravasation and in $11(21 \%)$ of 52 empirically embolized patients: Six (19\%) of 32 patients with pathologic arteries visible on aortography, $3(18 \%)$ of 17 with pathologic arteries visible by selective arteriography and $2(67 \%)$ of 3 with no visible pathologic arteries. From 6 patients $(11 \%, 5$ male, 1 female) who died within 30 days after embolization, 3 suffered from tuberculosis while 3 had malignant tumors. Three had ongoing hemoptysis. One patient died of multiple organ failure caused by post-interventional paraplegia and consecutive pneumonia. Con-
\end{abstract}

\footnotetext{
*The authors declared no conflicts of interest.
}

clusion: In patients with hemoptysis, empirical embolization is effective when pathologic bronchial arteries can be identified by DSA.

Keywords: Empiric Bronchial Arterial Embolization

\section{INTRODUCTION}

The resurgence of tuberculosis in industrialized western nations since the 1980s [1] has been the major factor to keep hemoptysis in daily clinical routine [2]. Aside this most common cause, the occurrence of hemoptysis in patients worldwide most often occurs in the setting of chronic inflammatory processes including other infectious diseases (such as aspergillosis) and noninfectious etiologies including neoplasms, cystic fibrosis and bronchiectasis [3].

About $1.5 \%$ of reported cases are categorized as "massive" or severe [4]. Massive hemoptysis is usually defined as an oral volume loss of more than $300 \mathrm{ml}$ in a 24 hour period $[5,6]$. It is a serious potentially life threatening condition, as bleeding into the tracheobronchial tree will inevitably occur, resulting in asphyxiation. Considering the dreary consequences, swift actions must be taken against hemorrhage resulting in compromise of the patient's pulmonary or hemodynamic status [7].

The large array of possible causes for hemoptysis provides a challenge for clinicians to evaluate and manage. Together with obligatory blood testing and initial chest radiography, bronchoscopy (especially rigid bronchoscopy) has proven to be an excellent diagnostic method for localization of the source of hemorrhage $[2,8]$. If there is time, multidetector computed tomography (CT) 
can give additional anatomical information-in particular on vascular malformations - which may improve consequent patient management [9].

In the past, surgery in an emergency setting and conservative therapy (i.e. medication and observation) have been related to mortality rates as high as $40 \%$ [10] and $50 \%$ [5], respectively. In our time, bronchial artery embolization (BAE) is the gold standard to control pulmonary hemorrhage in most cases with reported initial success rates of $70 \%-100 \%$ and one-year-success rates of $64 \%-82 \%[11,12]$. Whereas active bleeding can be effectively treated by embolization, the efficacy of empirical embolization is discussed. Empirical embolization is defined as endovascular occlusion of arteries without visible contrast-material extravasation on digital subtraction angiography. In the abdominal region it has already been shown to be safe and efficient when a bleeding site cannot be determined by angiography [13]. We conducted a retrospective review on patients with severe hemoptysis that have been treated by BAE to evaluate the efficacy of empiric arterial embolization in this setting.

\section{MATERIALS AND METHODS}

Histories as well as interventional reports of 58 patients (42 male, 16 female) referred for endovascular treatment of massive hemoptysis over a period of 17 years and 10 months (June 1995 to March 2013) were reviewed for data collection. Median age was 57.5 years (range, 16 83 years). Underlying diseases were tuberculosis in 21, malignant tumors in 24 (18 bronchial carcinoma, 2 patients with metastasis of esophageal cancer, 1 metastasis of laryngeal cancer, 1 metastasis of parotid cancer, 1 pleural mesothelioma, 1 plasmocytoma), bronchiectases in 11 and cystic fibrosis in 2 cases. The study was approved by the local ethics committee (Approval Number $25-058$ ex 12/13). All patients underwent fiberoptic bronchoscopy or CT angiography of the thorax before intervention. Using a fluoroscopy unit (Siemens, Erlangen, Germany), all interventions were performed via a transfemoral vascular access using a 6F sheath (Cordis Corporation, Bridgewater, NJ, USA). Initial thoracic aortography was carried out using a $6 \mathrm{~F}$ pigtail catheter (Cordis Corporation, Bridgewater, NJ, USA) to demarcate the size, number and position of the bronchial arteries. Iodinated contrast media $(300 \mathrm{mg} / \mathrm{ml})$ were used. If no bronchial arteries were detected on aortic overview, selective probing of thoracic aortic side-branches as well as the brachio-cephalic trunk and the subclavian arteries was intended by using different catheters. Consecutively, selective angiography of the bronchial arteries was performed using $4 \mathrm{~F}$ cobra, $4 \mathrm{~F}$ sidewinder or hinck headhunter catheters (Cordis Corporation, Bridgewater, NJ, USA; Terumo Medical, Somerset, NJ, USA; Boston Sci- entific, Natick, MA, USA). Arteries were regarded to be pathologic when they supplied an actively bleeding branch or an angiographic hypervascular section of the lung or were hypertrophic. All detected pathologic arteries that were supposed to supply the hemorrhagic bronchi seen on fiber-optic bronchoscopy were embolized with spherical embolization particles of $200-700 \mu \mathrm{m}$ in diameter. Superselective embolization was performed via a coaxial 0.018 inch microcatheter/wire system (FastTracker, Boston Scientific, Natick, MA, USA) until stasis occurred in the selected vessels. In the case of absence of visible pathologic bronchial or ectopic arteries, intercostal arteries supposed to supply the bleeding site via pleural adhesions were embolized. Technical success —reflecting immediate angiographic results [14]—was defined as complete perfusion-cessation in the embolized arteries. Clinical success was defined as the absence of ongoing hemoptysis within a period of 30 days after embolization [14]. Median follow up time was 46 months (range, 2 to 84 months). Figures 1(a), (b), 2(a) and (b) show typical examples of successful empiric arterial embolization. After gathering patient data, we analyzed the digital subtraction angiographic (DSA) studies with respect to the type of embolized arteries. The patients were categorized by underlying diseases, active bleeding (if absent, the intervention was defined as "empiric") and/ or visible pathologic arteries during angiography, success, and 30-day mortality.

\section{Statistical Evaluation}

The data was analyzed using descriptive statistics. For continuous data, median and range were reported and for categorical data frequencies and relative frequencies were reported.

\section{RESULTS}

In $33(57 \%)$ of the 58 patients pathologic arteries were detected on overview aortography. In $18(31 \%)$ patients pathologic arteries were detected by selective angiography. In $3(5 \%)$ patients no pathologic arteries were detected, with bronchial carcinoma being their underlying disease. Detected pathologic vessels were bronchial arteries in $29(50 \%)$, branches of intercostal arteries in 6 $(10 \%)$, branches of the intercostobronchial trunk in 7 $(12 \%)$, branches of the intercostobronchial trunk and of intercostal arteries in $6(10 \%)$, bronchial arteries and branches of the internal thoracic artery in $5(9 \%)$, bronchial arteries and branches of intercostal arteries and the internal thoracic artery in $5(9 \%)$ patients. Mean time of intervention was 73 minutes (range, 21 to 150 minutes). See Table 1 for a comparison of artery types and outcome.

In all patients $(100 \%)$ the target arteries could be em- 


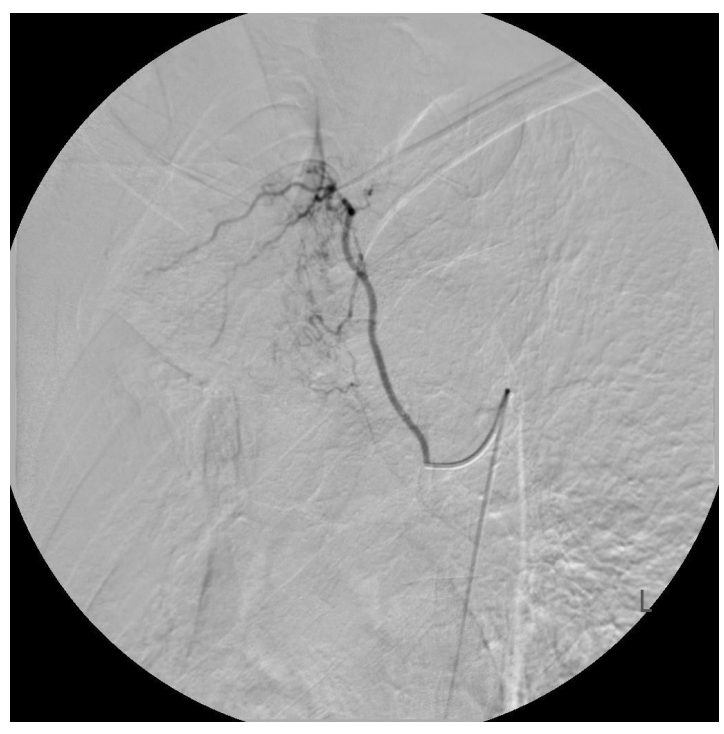

(a)

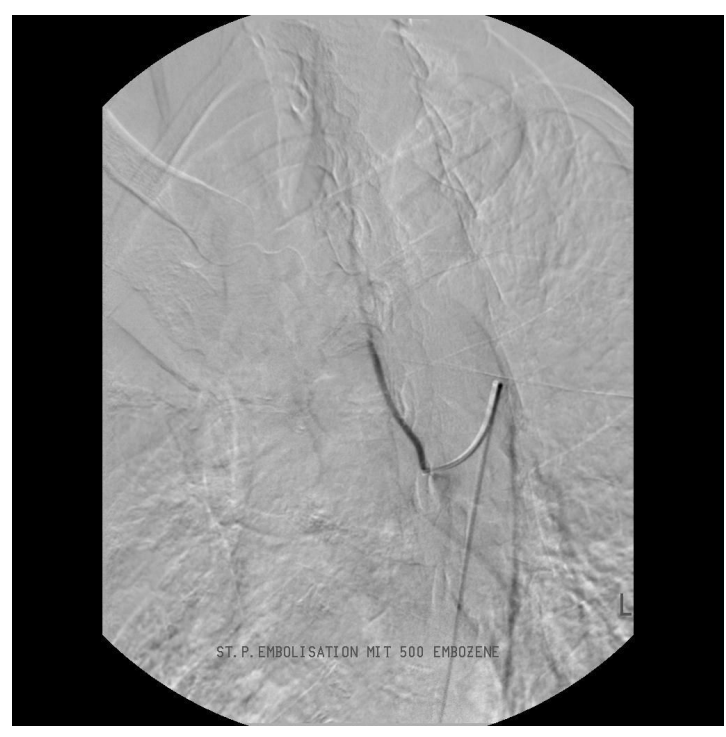

(b)

Figure 1. DSA in a 67-year-old patient with tuberculosis demonstrating a hypertrophic bronchial artery in the right upper lobe before and after embolization.

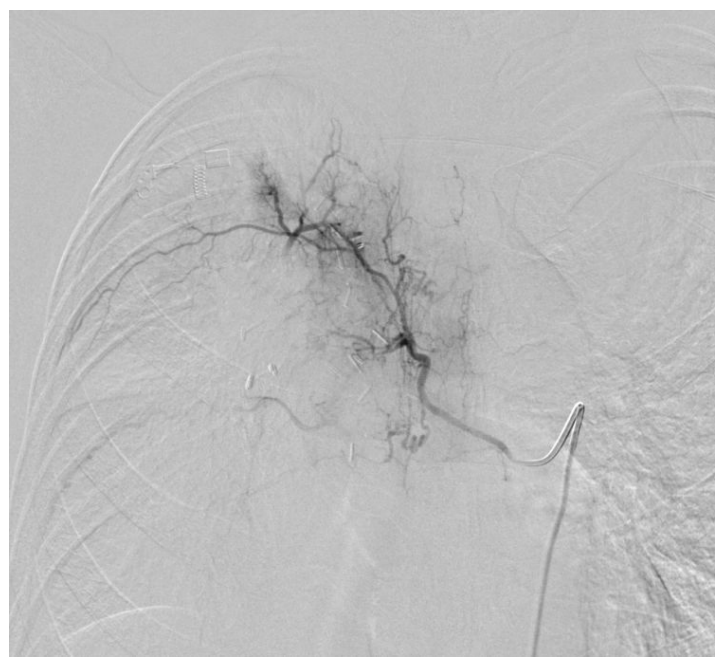

(a)

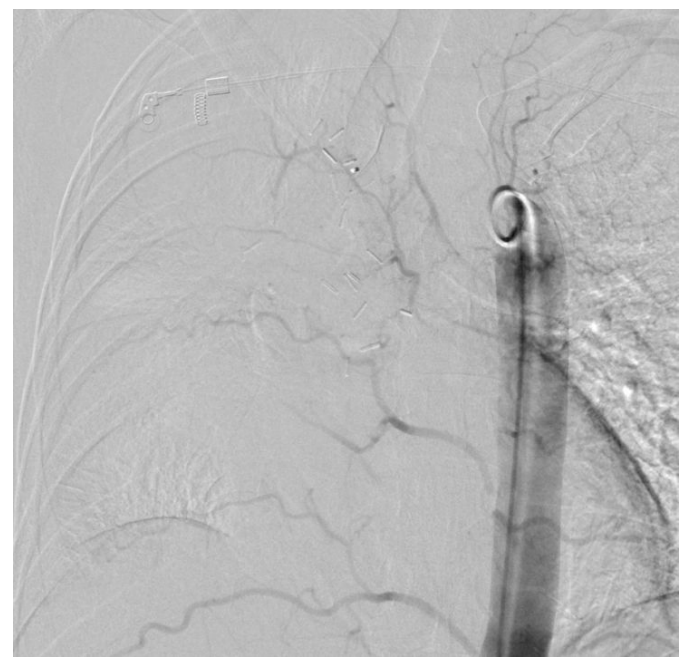

(b)

Figure 2. DSA in a 47-year-old patient with pulmonary metastases of parotid carcinoma demonstrating a hypertrophic bronchial artery in the right upper lobe before and after embolization.

Table 1. Type of artery and outcome (percentages are rounded to integer).

\begin{tabular}{|c|c|c|c|c|c|}
\hline \multicolumn{2}{|c|}{ Type of artery and outcome } & \multicolumn{3}{|c|}{ Empirical embolizations } & \multirow[b]{2}{*}{$\Sigma$} \\
\hline & $\begin{array}{l}\text { Acute extravasation } \\
\text { seen on fluoroscopy }\end{array}$ & $\begin{array}{l}\text { Pathologic bronchial } \\
\text { arteries detected on } \\
\text { aortography }\end{array}$ & $\begin{array}{c}\text { Pathologic bronchial arteries } \\
\text { detected on selective } \\
\text { angiography }\end{array}$ & $\begin{array}{c}\text { No pathologic } \\
\text { arteries detected }\end{array}$ & \\
\hline $\begin{array}{l}\text { Ongoing hemoptysis } \\
\text { ( }<30 \text { days) }\end{array}$ & 1 & 6 & 3 & 2 & $12(21 \%)$ \\
\hline $\begin{array}{l}\text { No ongoing } \\
\text { hemoptysis }\end{array}$ & 3 & 27 & 14 & 2 & $46(79 \%)$ \\
\hline$\Sigma$ & $4(7 \%)$ & $33(57 \%)$ & $17(29 \%)$ & $4(7 \%)$ & $58(100 \%)$ \\
\hline
\end{tabular}


bolized until perfusion-stasis. We discovered continuing hemoptysis in one $(25 \%)$ of 4 patients with active contrast extravasation. Also, in $11(20 \%)$ of 54 empirically embolized patients, no cessation of hemoptysis could be achieved within a period 30 days: $6(18 \%)$ of 33 patients with hypertrophic bronchial arteries visible on aortography, 3 (18\%) of 17 with hypertrophic arteries visible by selective arteriography and $2(50 \%)$ of 4 with no visible pathologic arteries, respectively. From 6 patients $(10 \%, 5$ male, 1 female) who died within 30 days after embolization, 3 suffered from tuberculosis while 3 had malignant tumors. Three of these 6 patients had ongoing hemoptysis, with one suffering from tuberculosis and two from malignant tumors. Major complications [14] occurred in one $(2 \%)$ patient who died of multiple organ failure caused by post-interventional paraplegia and consecutive pneumonia. The patient's underlying disease was tuberculosis. Two hypertrophic bronchial arteries were embolized until stasis in the right upper lobe without ongoing hemoptysis. No anterior spinal artery was detected during intervention.

\section{DISCUSSION}

Considered as gold standard, bronchial artery embolization (BAE) is the therapy of choice to control severe hemoptysis in the majority of cases $[7,9,12,151-18]$ We detected active extravasation in 7\% (four patients), compared with $10.7 \%$ reported in other studies [19].

In our study, continuous hemoptysis was reported in $20 \%$ of the patients who underwent empirical embolization and in $25 \%$ of the patients with active contrast extravasation. Thus, empirical embolization revealed to be as effective as embolization of visibly bleeding arteries.

Empiric embolization was successful in $82 \%$ of patients when pathologic arteries could be detected, no matter whether they were seen by overview or selective angiographies. However, the success rate decreased dramatically to $50 \%$ when no pathologic arteries were found.

The main reason why no pathologic arteries could be detected in 4 patients may lie in the existence of ectopic arteries that were not seen despite selective angiography of the brachiocephalic trunk and the subclavian arteries. The growth of ectopic arteries is frequently mediated by Vascular Endothelial Growth Factor expressed by tumors like non-small-cell lung carcinoma, for instance [20]. Anatomically, in $80 \%$ of the population bronchial arteries originate from the descending thoracic aorta, typically between the T5 and T6 vertebral bodies [21,22]. The remaining $20 \%$, however, do not arise as first order branches from the thoracic aorta but from other vessels, e.g. the subclavian, internal mammary or celiac trunk [22]. Furthermore, there are various anastomoses between the bronchial arteries and the pulmonary arteries at multiple levels [21]. Lastly, possible arteriovenous shunting can hamper a positive outcome. Another possible reason (although not presented in our trial, since all three patients' underlying disease was bronchial carcinoma) may be the pathophysiology of massive hemoptysis caused by chronic inflammation which is also a condition associated with cystic fibrosis, raising the lifetime hemoptysisrisk to $4.1 \%$ [23]. Chronic inflammation of the airways, possibly in combination with bacterial products from Staphylococcus aureus [24], causes angiogenesis and microvascular remodeling, stimulating bronchial artery hypertrophy and angiogenesis resulting in pathologic vessels with bronchopulmonary anastomoses and collaterals [25].

Since we discovered continuous hemoptysis in 2 of 4 patients with no visible pathologic arteries after the embolization of normal arteries, our study shows a lack of efficacy in BAE of these patients, in particular when non-pathologic intercostal arteries are embolized. Therefore, embolization of non-pathologic intercostal arteries is not advisable. Furthermore, embolization of intercostal arteries carries the risk of paraplegia [26].

We were able to control pulmonary hemorrhage and prevent ongoing hemoptysis by empiric BAE in $80 \%$, which sets the rate of clinical success within the range of initially reported rates $(70 \%-100 \%)(15-22)$, but lower than the suggested threshold of $85 \%$ (30). Early cessation of the search for pathologic arteries may contribute to the $20 \%$ of patients with ongoing hemoptysis. In some patients, no further look for ectopic arteries was done when distinct pathologic arteries had already been embolized.

Some limitations to this study have to be considered: First, the study was performed in a retrospective manner. Second, some treated patients might have been presented to other hospitals that could not be assessed by our interhospital medical documentation database. Consecutively, not all recurrent cases of hemoptysis may have been reported. Finally, the interventions were performed by five different interventionists whose algorithms may have varied to some extent.

In conclusion, our study shows that empiric BAE is efficient when hypertrophic bronchial arteries can be identified by DSA. Empiric embolization of nonpathologic arteries is not suggested; nonetheless the search for ectopic arteries is indicated at all times in order to rule out collateral vascularisation.

\section{REFERENCES}

[1] Faustini, A., Hall, A.J. and Perucci, C.A. (2006) Risk factors for multidrug resistant tuberculosis in Europe: A systematic review. Thorax, 61, 158-163. doi:10.1136/thx.2005.045963 
[2] Jean-Baptiste, E. (2000) Clinical assessment and management of massive hemoptysis. Critical Care Medicine, 28, 1642-1647. doi:10.1097/00003246-200005000-00066

[3] Hirshberg, B., Biran, I., Glazer, M., et al. (1997) Hemoptysis: Etiology, evaluation, and outcome in a tertiary referral hospital. Chest, 112, 440-444. doi: 10.1378/chest.112.2.440

[4] Wyngaarden, J., Smith, L. and Bennett, J. (1992) Cecil textbook of medicine. 19th Edition, WB Saunders, Philadelphia, 370.

[5] Crocco, J., Rooney, J., Fankushen, D., et al. (1968) Massive hemoptysis. Archives of Internal Medicine, 121, 495498. doi:10.1001/archinte.1968.03640060009002

[6] Ferris, E.J. (1981) Pulmonary hemorrhage. Vascular evaluation and interventional therapy. Chest, 80, 710-714. doi:10.1378/chest.80.6.710

[7] Sopko, D.R. and Smith, T.P. (2011) Bronchial artery embolization for hemoptysis. Seminars in Interventional $\mathrm{Ra}-$ diology, 28, 48-62. doi:10.1055/s-0031-1273940

[8] Dweik, R.A. and Stoller, J.K. (1999) Role of bronchoscopy in massive hemoptysis. Clinics in Chest Medicine, 20, 89-105. doi:10.1016/S0272-5231(05)70129-5

[9] Chun, J.-Y., Morgan, R. and Belli, A.-M. (2010) Radiological management of hemoptysis: A comprehensive review of diagnostic imaging and bronchial arterial embolization. Cardiovascular and Interventional Radiology, 33, 240-250. doi:10.1007/s00270-009-9788-Z

[10] Fernando, H.C., Stein, M., Benfield, J.R., et al. (1998) Role of bronchial artery embolization in the management of hemoptysis. Archives of Surgery, 133, 862-866. doi:10.1001/archsurg.133.8.862

[11] Cohen, A.M., Doershuk, C.F. and Stern, R.C. (1990) Bronchial artery embolization to control hemoptysis in cystic fibrosis. Radiology, 175, 401-405.

[12] Uflacker, R., Kaemmerer, A., Picon, P.D., et al. (1985) Bronchial artery embolization in the management of hemoptysis: Technical aspects and long-term results. Radiology, 157, 637-644.

[13] Walsh, R.M., Anain, P., Geisinger, M., et al. (1999) Role of angiography and embolization for massive gastroduodenal hemorrhage. Journal of Gastrointestinal Surgery, 3, 61-65.

[14] Angle, J.F., Siddiqi, N.H., Wallace, M.J., et al. (2010) Quality improvement guidelines for percutaneous transcatheter embolization: Society of Interventional Radiology Standards of Practice Committee. Journal of Vascular and Interventional Radiology, 21, 1479-1486. doi:10.1016/j.jvir.2010.06.014
[15] Kalva, S.P. (2009) Bronchial artery embolization. Techniques in Vascular and Interventional Radiology, 12, 130138. doi:10.1053/i.tvir.2009.08.006

[16] Chawla, R.K., Madan, A., Mehta, D., et al. (2010) Controlling hemoptysis: An alternative approach. Lung India, 27, 99-101. doi:10.4103/0970-2113.63616

[17] Uflacker, R., Kaemmerer, A., Neves, C., et al. (1983) Management of massive hemoptysis by bronchial artery embolization. Radiology, 146, 627-634.

[18] Jougon, J., Ballester, M., Delcambre, F., et al. (2002) Massive hemoptysis: What place for medical and surgical treatment. European Journal Cardio-Thoracic Surgery, 22, 345-351. doi:10.1016/S1010-7940(02)00337-8

[19] Ramakantan, R., Bandekar, V.G., Gandhi, M.S., et al. (1996) Massive hemoptysis due to pulmonary tuberculosis: Control with bronchial artery embolization. Radiology, 200, 691-694.

[20] Jackson, A.L., Zhou, B. and Kim, W.Y. (2010) HIF, hypoxia and the role of angiogenesis in non-small cell lung cancer. Expert Opinion on Therapeutic Targets, 14, 1047 1057. doi:10.1517/14728222.2010.511617

[21] Pump, K.K. (1972) Distribution of bronchial arteries in the human lung. Chest, 62, 447-451. doi:10.1378/chest.62.4.447

[22] Stoll, J.F. and Bettmann, M.A. (1988) Bronchial artery embolization to control hemoptysis: A review. Journal of Vascular and Interventional Radiology, 11, 263-269. doi:10.1007/BF02577032

[23] Flume, P.A., Yankaskas, J.R., Ebeling, M., et al. (2005) Massive hemoptysis in cystic fibrosis. Chest, 128, 729738. doi:10.1378/chest.128.2.729

[24] Boussaud, V., Parrot, A., Mayaud, C., et al. (2003) Lifethreatening hemoptysis in adults with community-acquired pneumonia due to Panton-Valentine leukocidin-secreting Staphylococcus aureus. Intensive Care Medicine, 29, 1840-1843. doi:10.1007/s00134-003-1918-5

[25] McColley, S.A., Stellmach, V., Boas, S.R., et al. (2000) Serum vascular endothelial growth factor is elevated in cystic fibrosis and decreases with treatment of acute pulmonary exacerbation. American Journal of Respiratory and Critical Care Medicine, 161, 1877-1880. doi:10.1164/ajrccm.161.6.9905022

[26] Cowling, M.G. and Belli, A.M. (1995) A potential pitfall in bronchial artery embolization. Clinical Radiology, 50, 105 107. doi:10.1016/S0009-9260(05)82990-3 